\title{
A Power-Saving Modulation Technique for Time-of-Flight Range Imaging Sensors
}

\author{
Richard M. Conroy*, Adrian A. Dorrington, Andrew D. Payne, \\ Rainer Künnemeyer and Michael J. Cree. \\ School of Engineering, The University of Waikato, \\ Private Bag 3105, Hamilton, New Zealand.
}

\begin{abstract}
Time-of-flight range imaging cameras measure distance and intensity simultaneously for every pixel in an image. With the continued advancement of the technology, a wide variety of new depth sensing applications are emerging; however a number of these potential applications have stringent electrical power constraints that are difficult to meet with the current state-of-the-art systems. Sensor gain modulation contributes a significant proportion of the total image sensor power consumption, and as higher spatial resolution range image sensors operating at higher modulation frequencies (to achieve better measurement precision) are developed, this proportion is likely to increase. The authors have developed a new sensor modulation technique using resonant circuit concepts that is more power efficient than the standard mode of operation. With a proof of principle system, a 93-96\% reduction in modulation drive power was demonstrated across a range of modulation frequencies from 1-11 MHz. Finally, an evaluation of the range imaging performance revealed an improvement in measurement linearity in the resonant configuration due primarily to the more sinusoidal shape of the resonant electrical waveforms, while the average precision values were comparable between the standard and resonant operating modes.
\end{abstract}

Keywords: Time-of-flight, range imaging, pixel modulation, PMD, power-saving, resonant circuit

\section{INTRODUCTION}

Time-of-flight range imaging cameras are a rapidly maturing depth sensing technology that is enabling a wide variety of new applications in areas such as automotive safety, natural user interfaces and medical imaging. These systems determine both distance and intensity simultaneously for every pixel in an image. Typically an amplitude modulated continuous wave $(\mathrm{AMCW})$ approach is employed using an active light source to illuminate the scene with a gain modulated image sensor to measure distance from the back-scattered light signal. The technology is gaining in prevalence due to a number of factors such as compactness, relative low-cost, parallel range acquisition nature, and most importantly, versatility. Systems can be configured to deliver depth images at video frame rates, or perform slower high-precision acquisitions, while the operating range, field of view and other system parameters can be customised to suit a given application.

For a number of new and emerging range imaging applications the power consumption of current time-of-flight cameras is a major limitation. Of particular concern are those where the system must operate on a limited power supply like battery or USB. Examples of such applications include mobile-phone, tablet or laptop integration, hand-held 3-D scanners, and mobile robotics. To compound the matter, as advancements are made to the camera technology such as increased spatial resolution and improved measurement performance, the required power is likely to increase. Accordingly, there is a desire to develop range imaging cameras that consume less power whilst not compromising functionality or performance.

This paper presents a practical means of reducing the power dissipation of a range imaging camera. Firstly, the various major power consuming hardware components are considered with a particular focus on the image sensor gain modulation sub-system. This is followed by the description of a new power-saving modulation mechanism that utilises resonant circuit concepts. A proof of principle system is then detailed along with an empirical evaluation of its electrical response (modulation signal and power measurements) in both standard and power-saving operating modes. Finally, a comparison of the range imaging performance between the two configurations is presented in terms of both measurement accuracy and precision.

*rmc21@waikato.ac.nz; eng.waikato.ac.nz

Videometrics, Range Imaging, and Applications XI, edited by Fabio Remondino, Mark R. Shortis, Proc. of SPIE Vol. 8085, 808506 - (C) 2011 SPIE · CCC code: 0277-786X/11/\$18 · doi: 10.1117/12.889729 


\section{TIME-OF-FLIGHT RANGE IMAGING}

AMCW time-of-flight range imaging cameras measure distance by determining the propagation delay of light emanating from the camera that is reflected back by objects in the scene of interest. The illumination source, normally an array of laser diodes or light emitting diodes (LEDs), is amplitude modulated, therefore the range-dependent propagation delay causes a phase shift in the modulation envelope of the back-scattered light.

To measure the phase shift, amplitude (active brightness), and DC offset of the reflected illumination a specialised image sensor is used where the pixels are gain modulated (during image integration) at the same frequency as the light source, typically in the range of 10-100 MHz. The detected photocurrent, representing the modulation envelope of the received light, is mixed with the sensor modulation waveform producing a correlation measurement at each pixel. In order to determine the unknown parameters independently of factors such as object reflectivity, intensity decay with distance, and ambient light, multiple correlation measurements are necessary. The most common approach is to capture four samples, $I_{0}$ to $I_{3}$, with the relative phase between the two modulation signals advanced electronically by $\pi / 2 \mathrm{rad}$ for each measurement. Using this configuration the amplitude, $A$, offset, $B$, and phase, $\varphi$, values are calculated as ${ }^{1}$

$$
\begin{gathered}
A=\frac{1}{2} \sqrt{\left(I_{0}-I_{2}\right)^{2}+\left(I_{1}-I_{3}\right)^{2}}, \\
B=\frac{1}{4} \sum_{k=0}^{3} I_{k}, \\
\varphi=\arctan \left(\frac{I_{0}-I_{2}}{I_{1}-I_{3}}\right) .
\end{gathered}
$$

Given the measured phase shift, $\varphi$, the speed of light, $c$, and the modulation frequency, $f_{m}$, the range, $r$, for each pixel can be calculated as a fraction of the modulation wavelength by

$$
r=\frac{\varphi c}{4 \pi f_{m}}
$$

\subsection{Measurement Linearity and Precision}

The accuracy to which phase (and therefore distance) can be determined is influenced by a number of factors, both external and internal to the camera, such as multi-path interference and temperature drift. One of the main sources of inaccuracy considered in this paper is the systematic measurement non-linearity which can occur depending on the shape of the illumination and sensor modulation waveforms. Equation 3 assumes that samples are taken from a sinusoidal correlation waveform, which is produced when at least one of the contributing modulation signals is sinusoidal. ${ }^{2}$ However, in practice it is common to use digital (square) modulation as the sensor and illumination system responses are usually non-linear and generating sinusoidal modulation is difficult. Unfortunately, during the sampling process harmonics present in the square modulation signals are aliased down onto the fundamental frequency, thus contaminating the phase measurement results with a cyclic linearity error. For a four sample configuration using ideal square illumination and sensor modulation, the expected peak-to-peak linearity error would be approximately $150 \mathrm{mrad}^{2}$

Another key performance metric that is predominantly influenced by the modulation signals is phase measurement precision (repeatability), defined as the standard deviation of measured phase values. In an ideal shot-noise limited system the phase precision, $\sigma_{\varphi}$, is given by ${ }^{3}$

$$
\sigma_{\varphi}=\frac{\sqrt{B / 2}}{c_{d} B_{s i g}},
$$

where $B_{\text {sig }}$ is the mean value of the detected active illumination and $B$ the total measured DC offset (given by equation 2), which is the sum of $B_{s i g}$ and any additional offset due to ambient light. The demodulation contrast, denoted $c_{d}$, is calculated as the ratio of the measured signal amplitude to signal offset $\left(A / B_{\text {sig }}\right)$ and gives a measure of the efficiency of the illumination and sensor gain modulation. Like the measured phase values, the amplitude $A$ is also influenced by 
modulation signal harmonics; so for non-sinusoidal modulation, the demodulation contrast and precision become phase dependent. $^{2}$

Finally, distance standard deviation, $\sigma_{r}$, is determined by scaling the phase precision, $\sigma_{\varphi}$, by $c /\left(4 \pi f_{m}\right)$ (analogous to equation 4). This indicates that range precision improves as the modulation frequency is increased, although in practice the bandwidth constraints of the light source and sensor modulation electronics impose an upper bound to this trend.

\section{OVERVIEW OF CAMERA POWER CONSUMPTION}

The power consumption of time-of-flight range imaging cameras varies considerably depending on the hardware configuration. While some leading manufacturers offer "one-size-fits-all" cameras for development purposes, typically a system is custom designed to suit a given application. Many system parameters can be tailored with relative ease, for example light source power and modulation frequency. However, altering fundamental features associated with the sensor, such as spatial resolution, requires considerable development.

Given the wide variation in how systems are configured, it is difficult to adequately quantify the major power contributions in general terms. Instead a brief outline is offered of the various factors that influence the power consumption of the following key system components:

- Pixel array and associated electronics.

- Analogue-to-digital (ADC) conversion and readout circuitry.

- Control and processing electronics.

- Light source and associated optics.

- Sensor modulation circuitry.

Pixel array power consumption is ultimately determined by spatial resolution, internal pixel electronics and associated peripheral circuits, while ADC power is not only dependent on the type of ADC used but also the arrangement. For example, a system designed for high readout speed might employ multiple ADCs in parallel but would consume more power than a single pipelined ADC approach.

The choice of control and processing electronics, which primarily determines the level of power consumption, depends on the required degree of functionality and range processing sophistication. Typical hardware consists of a field programmable gate array (FPGA), complex programmable logic device (CPLD), or digital signal processor (DSP), however a more power efficient approach is to integrate the necessary processing and control circuitry onto a custom ASIC (application specific integrated circuit) or onto the sensor itself.

Modulated light sources are designed to deliver the level of optical power required to measure to a specified maximum distance (considering the return path light intensity decay) for a given field of view and object reflectivity. Through the use of optics, light sources can be optimised by ensuring that only the camera field of view is illuminated, thereby minimising wasted light. Other factors to consider include the type of technology (lasers or LEDs), wavelength and modulation frequency. The amount of electrical power required is predominantly governed by the desired optical power, the choice of light source and the efficiency of the driving electronics.

Finally, of particular interest is the power consumed by the sensor modulation electronics. In traditional configurations, power depends on a number of factors such as modulation frequency and spatial resolution, and as technology improves power levels are likely to increase. The following section (3.1) examines sensor modulation power consumption in more detail. 


\subsection{Sensor Modulation Power Modeling}

In most range imaging sensor implementations, a specialised "two-tap" pixel structure is employed where each pixel consists of dual integration and storage regions ${ }^{1,4,5}$ (distinct from normal image sensors that have one storage region per pixel). Complimentary digital signals $\left(180^{\circ}\right.$ relative phase) drive two capacitive modulation gates that instruct the pixel to accumulate the detected light alternately in the two storage regions. Frequencies used for sensor modulation traditionally lie in the range of $10-100 \mathrm{MHz}$.

For power analysis purposes, an individual pixel modulation gate can be modeled as a small capacitor $\left(C_{p i x}\right)$, with the full pixel array considered as two separate networks of these capacitors connected in parallel. A typical layout of the sensor modulation network is shown in figure 1(a) where modulation lines A and B are sourced from the top of the array and run column-wise down the sensor. While it is not shown here, in some embodiments a distributed driver arrangement is used where individual digital buffers drive each column, or banks of columns. Figure 1(b) zooms in on an individual pixel showing a simplified model of the two modulation gate "capacitors" connected to the resistive signal wiring. Depending on the level of resistance, the resulting distributed RC line can distort the shape of the square electrical modulation waveform as it propagates down the column.

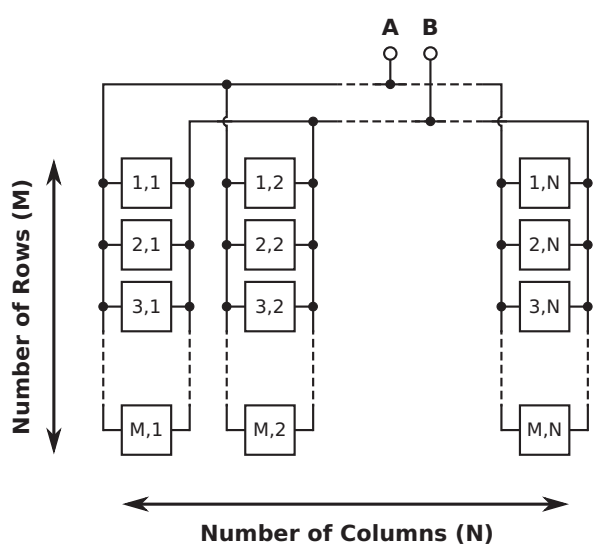

(a) Pixel array layout

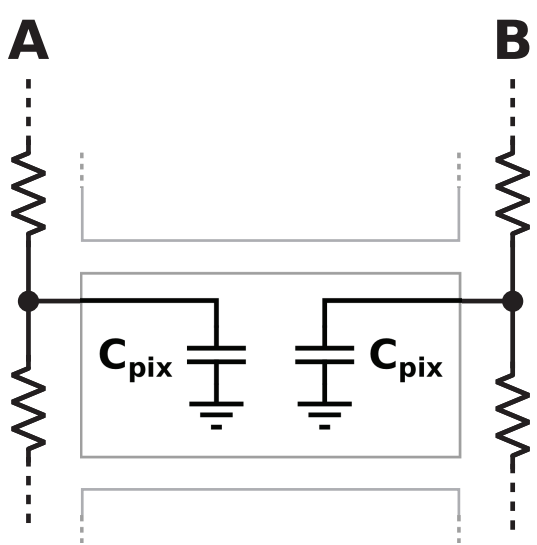

(b) Pixel model

Figure 1. (a) High-level layout of a typical "two-tap" pixel array with modulation signals (A and B) running column-wise down the sensor, (b) a simplified electrical model of an individual pixel illustrating the capacitive modulation gates and resistive signal wiring.

Power is consumed during the charging and discharging of the two capacitive networks. The total average power consumption can be calculated using

$$
P=2 M N C_{p i x} V^{2} f_{m},
$$

where $M$ and $N$ are the number of rows and columns respectively, $f_{m}$ is the modulation frequency, and $V$ is the square wave voltage amplitude. The product $2 M N C_{p i x}$ represents the total cumulative modulation gate capacitance of the entire sensor.

With technology advancing and applications demanding higher spatial resolution and improved measurement performance (often achieved using higher modulation frequencies), the power consumption due to sensor modulation is becoming an increasingly dominant proportion of the total system power. This is evident in equation 6 with power scaling linearly with both frequency and the number of pixels $(M \times N)$. Also of concern is that high-current spikes generated due to digital switching can lead to transient power supply disturbance which may cause significant interference with other sensitive parts of the system.

Consider a realistic example using parameters that are comparable to those of some current state-of-the-art systems. Given a QVGA resolution sensor $(320 \times 240$ pixels $)$ with a modest modulation gate capacitance of $C_{\text {pix }}=20 \mathrm{fF}$, the modulation drive circuit will consume $3.3 \mathrm{~W}$ when operating at a voltage of $3.3 \mathrm{~V}$ and a frequency of $100 \mathrm{MHz}$. To offer some perspective, this level of power consumption would likely be prohibitive if such a camera were to be powered by battery or USB ( $2.5 \mathrm{~W}$ power limit), especially considering this figure does not include the power consumption of other major system components. 


\section{POWER SAVING SENSOR MODULATION}

When considering ways to save power in range imaging cameras, the sensor modulation sub-system is an obvious area to focus on. Ideally the principle requirement of any given power-saving approach is that there be no significant negative impact on range imaging performance, and this is the assumption used in this paper. However it is recognised that in some applications, a reduction in performance may be acceptable if significant power savings can be achieved.

\subsection{Possible Power Reduction Strategies}

There are a number of possible strategies that could be employed to help reduce sensor modulation power. An obvious approach is to reduce the capacitance of the sensor array by developing pixels with a lower modulation gate capacitance $\left(C_{p i x}\right)$. However, based on current technology it would be difficult to achieve any appreciable reduction in capacitance without compromising pixel functionality and performance, or encountering IC process limitations (design rules). Moving away from traditional range imaging sensor structures, new low-capacitance sensor designs may be possible, although the authors are unaware of any such developments.

A relatively simple power saving technique is to reduce the voltage amplitude of the square modulation signal. With $P \propto V^{2}$ (from equation 6), lowering the voltage by 50\% will result in a 75\% reduction in power consumption. While there may be some technologies where this approach could work, in general there are key reasons why it would not be suitable. Most importantly, weaker electric fields within the pixel could adversely affect performance and functionality; for example, the PhotonICs ${ }^{\circledR}$ PMD 19k time-of-flight sensor from PMD Technologies GmbH (Siegen, Germany) exhibits a drop in demodulation contrast from $32 \%$ to $12 \%$ when the modulation voltage is halved from $2 \mathrm{~V}$ to $1 \mathrm{~V},{ }^{6}$ which in practice would mean poorer measurement precision (from equation 5).

In a differential system, charge sharing can be used to save power. This technique works by shorting the two oppositepolarity pixel modulation lines (A and B) prior to the high-to-low (or low-to-high) voltage transition, which temporarily brings both capacitive gates to a mid-supply voltage. Consequentially, less energy is required by the driver to complete the transition thus reducing the power consumed. Theoretically, 50\% power savings can be achieved using this technique, although with resistive losses in the signal wiring, actual savings may be considerably smaller depending on the physical circuit layout.

Finally, a resonant LC tank circuit can be used to more efficiently modulate the image sensor by incorporating an inductor with the modulation electronics. The inductor resonates with the capacitive array of pixels setting up a sinusoidallike modulation waveform that requires a relatively small amount of energy to maintain. In the field of high density, high frequency digital electronics where the power consumption due to digital clocking is substantial, various "resonant clocking" implementations have been demonstrated. ${ }^{7-11}$ In one particular case an $84 \%$ reduction was achieved ${ }^{11}$ with the level of savings dependent on the losses in the resistive wiring network and inductor quality. For range imaging, resonant modulation potentially offers large power savings with minimal impact on measurement performance.

\subsection{Resonant Modulation for Range Imaging}

When an inductor is connected across a charged capacitor, energy is repeatedly transferred between the electric field of the capacitor and the magnetic field of the inductor. The voltage across the capacitor (capacitance $C$ ) oscillates sinusoidally as current flows back and forward through the inductor (inductance $L$ ) at the natural resonant frequency $\left(f_{\text {res }}\right)$ given by

$$
f_{\text {res }}=\frac{1}{2 \pi \sqrt{L C}} \cdot \sqrt{1-\zeta^{2}},
$$

where $\zeta$ is a damping factor that depends largely on the resistive losses in the circuit. Formulae for damping factor vary depending on the resonant circuit configuration.

In a range imaging context, given the typical differential pixel structure used in most state-of-the-art sensors (i.e. two modulation gates per pixel), a differential resonant circuit is the most practical configuration. A highly simplified schematic of the implementation is shown in figure 2(a). In this circuit energy is effectively recycled by pushing charge back and forth between the two capacitors $C_{A}$ and $C_{B}$. 


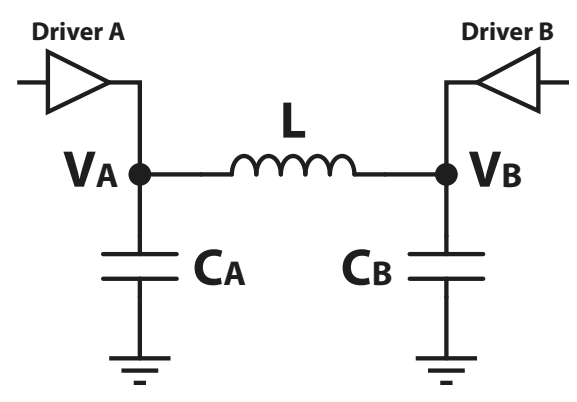

(a) Schematic

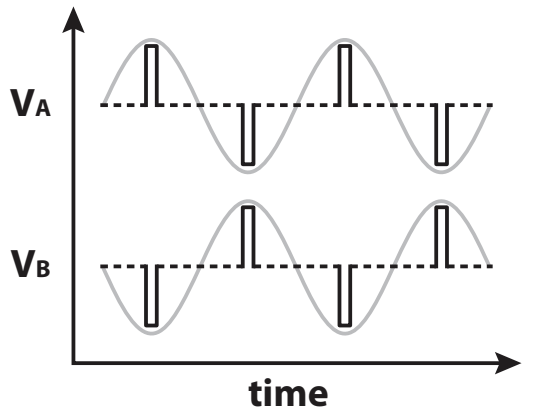

(b) Waveforms

Figure 2. (a) Simplified differential LC tank circuit and (b) modulation waveforms, where the dark lines show the unloaded driver outputs with broken lines representing the drivers in a high-impedance output state, while the light sinusoidal shaped lines show the resulting pixel modulation signals in the resonant configuration.

These capacitors $\left(C_{A}\right.$ and $\left.C_{B}\right)$ are lumped representations of the two distributed networks of pixel modulation gates (refer to figure 1), where $C_{A}=C_{B}=M N C_{p i x}$. In this particular circuit configuration, the inductance $L$ for a desired resonant frequency can be approximated by (assuming $\zeta=0$ )

$$
L \approx \frac{2}{C_{A}\left(2 \pi f_{r e s}\right)^{2}} .
$$

In a lossless resonant circuit, zero power is consumed as all energy is recycled and oscillations are sustained indefinitely. However, in practical implementations some of the energy is dissipated in the resistive wiring of the circuit and lost due to leakage and inefficiencies in the components resulting in damped oscillations that eventually die away $(\zeta>0)$. To sustain the resonant waveform the lost energy needs to be restored, thereby consuming some power.

In our configuration two tri-state driver circuits, Driver $A$ and Driver $B$, are used to replenish the resonant circuit. An illustration of the circuit operation is shown in figure 2(b) with the dark waveform representing the unloaded driver output. Firstly nodes $V_{A}$ and $V_{B}$ are pulsed to high and low voltages respectively, set to high-impedance (broken line) and left to oscillate at the natural resonant frequency. The driver then pulses the nodes to the opposite polarity before leaving to oscillate again, at which point the cycle is repeated.

When the driving repetition rate is matched to the resonant frequency, power consumption is minimised and two sinusoidal-like modulation waveforms with a relative phase of $180^{\circ}$ will result (light coloured waveforms in figure 2(b)), although the precise shape will depend largely on the resistive losses in the circuit. Another important parameter is the pulse width of the driver signal, which needs to be wide enough to sufficiently replenish the tank circuit while not exceeding the point of magnetic saturation and dissipating energy unnecessarily.

\subsection{PMD Implementation}

The full benefits of resonant modulation will ultimately be realised once fully integrated on a range imaging sensor, but as an initial step the authors have demonstrated a proof of principle implementation using discrete components. Previously the authors developed a range imaging camera ${ }^{12}$ using a PhotonICs ${ }^{\circledR}$ PMD 3k-S time-of-flight image sensor (PMD Technologies GmbH, Siegen, Germany). The system consists of a custom PCB that houses the sensor and all associated control, modulation and readout electronics, interfaced to an FPGA which provides overall control of the camera. The PMD 3k-S sensor is ideal for investigating resonant modulation as it provides direct uninhibited access to the capacitive modulation network via package pins (with no on-chip modulation electronics or ESD protection circuitry). ${ }^{5}$ With some minor hardware modifications the original PMD system has been adapted so that it can operate in the resonant configuration.

A simplified schematic of the PMD resonant modulation circuitry is shown in figure 3. The PMD $3 \mathrm{k}-\mathrm{S}$ sensor has an effective imaging resolution of $64 \times 48$ pixels, although with two additional dummy rows for compensation the actual load seen by the modulation drivers is $64 \times 50$ pixels. Each modulation gate (two per pixel) has a nominal capacitance of $C_{\text {pix }}=0.5 \mathrm{pF}$ resulting in a total load of $1.6 \mathrm{nF}$ per channel $\left(C_{A} \& C_{B}\right)$ over the entire sensor. To ease the loading 
on the driving electronics, the sensor is split into four vertically divided $16 \times 50$ pixel modulation regions, however in our implementation a single driver was used for each channel. This is depicted in the schematic where the output lines of the two drivers $\left(V_{A}\right.$ and $V_{B}$ ) fan out to the eight modulation pins (four per channel) of the PMD 3k-S sensor.

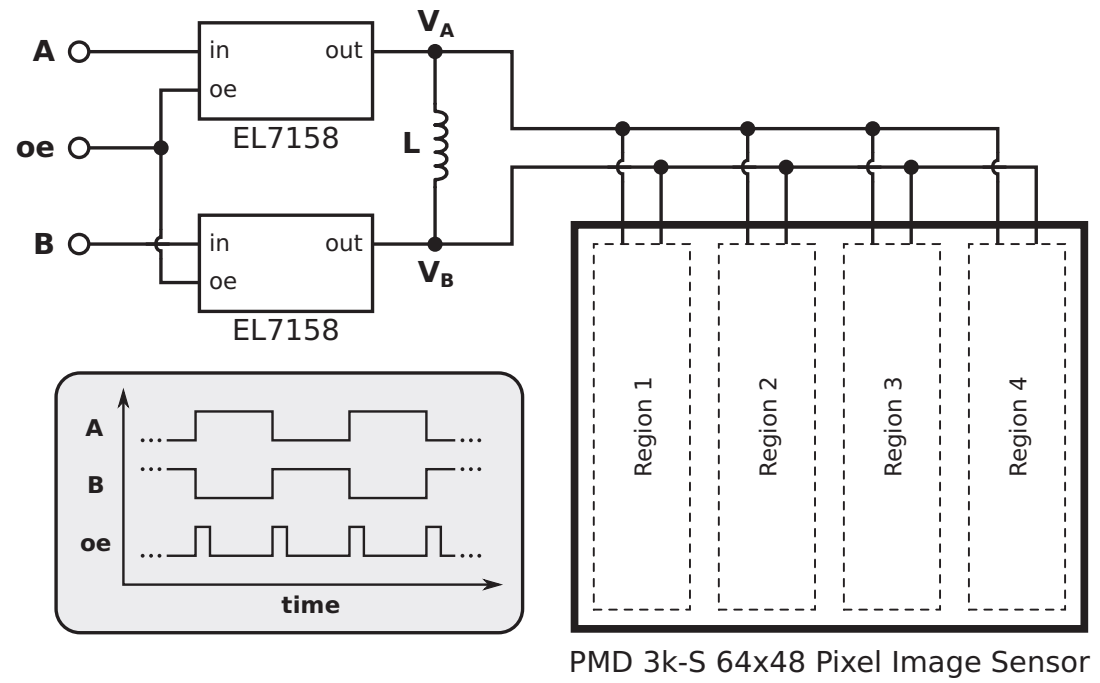

Figure 3. Schematic diagram showing the PMD resonant modulation driver circuitry, as well as representative digital input waveforms.

Sensor modulation is provided by two EL1758 tri-state pin drivers (Intersil Corp., Milpitas, CA, USA). The EL7158 is suitable for a traditional digital modulation configuration as it is designed to drive high capacitive loads at frequencies up to $40 \mathrm{MHz} .{ }^{13}$ Previous work using this PMD system has demonstrated optimum range imaging performance at $45 \mathrm{MHz} .{ }^{14}$ One feature of this driver is that its output can be set to a high-impedance state, so by appropriately pulsing the outputenable (oe) pin as illustrated in figure 3 the system can operate in a resonant configuration. In this mode, bandwidth limitations of the driver output-enable function constrain the maximum resonant operating frequency to around $11 \mathrm{MHz}$. While this relatively low frequency range is not ideal, it still offers sufficient scope for evaluating the resonant modulation principle in terms of electrical response and range imaging performance. In order to compare operation with traditional square modulation, the inductor was simply removed and the output-enable signal was held high.

With experiments to be conducted across a variety of operating frequencies, an assortment of discrete inductors were selected (using equation 8) such that their predicted resonant frequencies fall roughly within the range of 1-11 MHz. Altogether, eight inductors were chosen ranging from $33 \mu \mathrm{H}$ to $330 \mathrm{nH}$, each with very low DC resistance (95-750 m $\Omega$ ). The hardware was configured so that these inductors could be easily added and removed from the system between the outputs of the two EL7158 drivers. One concern with this external inductor configuration was whether parasitic bondwire inductance on the PMD sensor would influence the resonant circuit, however given the relatively large inductor sizes and low frequency operating range, any effect is considered negligible.

An Altera Stratix II FPGA was used to control all aspects of the PMD system, providing all sensor clocks, ADC readout, PC communications and modulation signal generation for both the sensor and light source. Compared to standard operation, the resonant configuration demands more complexity in the sensor modulation logic with the addition of the short duty-cycle driver output-enable signal. Also important is the ability to precisely control the modulation frequency in order to operate at resonance. All modulation signals are generated using an advanced phase locked loop (PLL) internal to the FPGA that offers the ability to dynamically configure the frequency, duty-cycle, and relative phase of its outputs.

\section{ELECTRICAL MEASUREMENT RESULTS}

Due to limited knowledge of the internals of the PMD 3k-S sensor, it is difficult to adequately predict the electrical response of the system in the resonant configuration through simulation. In order to compare the two operating modes, a series of measurements were recorded to determine the shape of the electrical modulation waveforms and the power consumed. 
All electrical sensor modulation measurements were performed using a Tektronix MSO4054 mixed signal oscilloscope. Sensor modulation power was determined using a small sense resistor (4.7 $\Omega$ ) placed in the main supply path of each EL7158 driver. By measuring the transient voltage across each resistor, the drive current, and therefore power, could be derived. In an effort to reduce noise susceptibility, these current sense signals were driven directly into $50 \Omega$ oscilloscope inputs. The two sensor modulation waveforms $\left(V_{A}\right.$ and $\left.V_{B}\right)$ were measured using high-impedance voltage probes at a point close to where the signals enter the PMD 3k-S sensor. An output enable pulse width of $15 \mathrm{~ns}$ was found to be optimum in all configurations using the EL7158 driver, so for consistency this value was used for all resonant modulation measurements reported herein.

\subsection{Finding the Resonant Frequency}

For resonant modulation optimum power savings are achieved when the driving frequency is matched to the natural resonant frequency of the tank circuit. This operating point was found empirically for each inductor by sweeping the driver modulation frequency and examining the resulting power measurements. The results of a frequency sweep using a $4.7 \mu \mathrm{H}$ inductor are shown in figure 4. In this case the optimum operating frequency was deemed to be $3.10 \mathrm{MHz}$, considerably higher than the predicted resonant frequency of $2.60 \mathrm{MHz}$. This mismatch of a higher than expected resonant frequency was observed across all test cases and is discussed further in section 5.3.

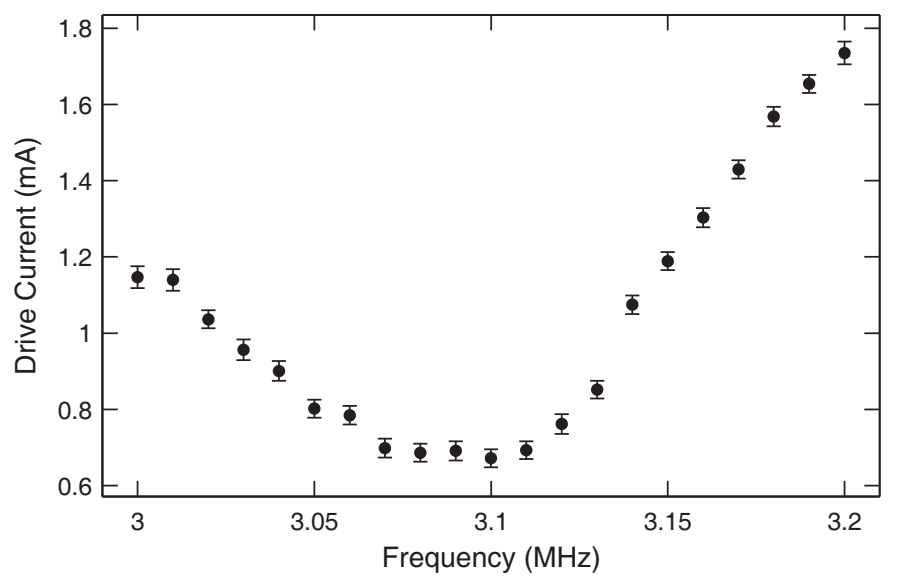

Figure 4. Measured average resonant modulation drive currents (using a $4.7 \mu \mathrm{H}$ inductor) for an operating frequency sweep showing that optimum power savings are achieved at roughly $3.10 \mathrm{MHz}$.

\subsection{Modulation Signal and Power Measurements}

The measured electrical modulation waveforms and drive current signals at $3.10 \mathrm{MHz}$ for both the standard and resonant (using the $4.7 \mu \mathrm{H}$ inductor) configurations are shown in figure 5. As expected the resonant modulation waveform has a sinusoidal shape with a peak-to-peak amplitude similar to that of the standard square waveform. From these electrical results it is difficult to predict the impact on range imaging performance with any certainty; although the obvious attenuation of higher order harmonics and perceived smaller fundamental frequency amplitude of the resonant signals would imply better linearity, yet poorer precision.

With the large current spikes evident in figure 5(b) significantly reduced in figure 5(d), it is clear that the average drive current using resonant modulation is considerably smaller than in the standard mode. The standard mode total average current was $16.72 \pm 0.04 \mathrm{~mA}$, while the resonant mode current was $0.67 \pm 0.02 \mathrm{~mA}$, meaning a power saving of $96 \%$ in this particular case. This level of savings was consistent across all other test cases where a 93-96\% reduction in modulation drive power was observed over a range of frequencies from 1-11 MHz. These results are illustrated in figure 6, which shows the total average modulation drive current versus frequency for both operating modes. Closer inspection of the standard modulation results reveals that, although drive current trends linearly with frequency as expected, the measured and theoretical values are not in agreement. This disparity is discussed in section 5.3. 


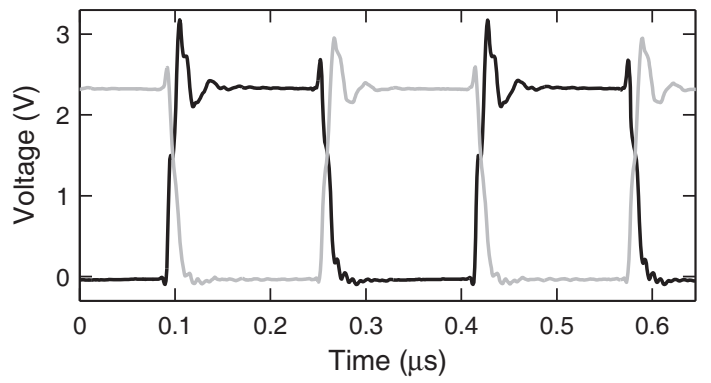

(a) Standard A \& B modulation waveforms

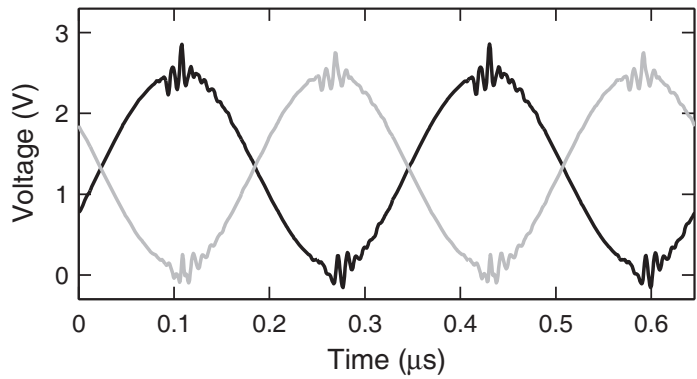

(c) Resonant A \& B modulation waveforms

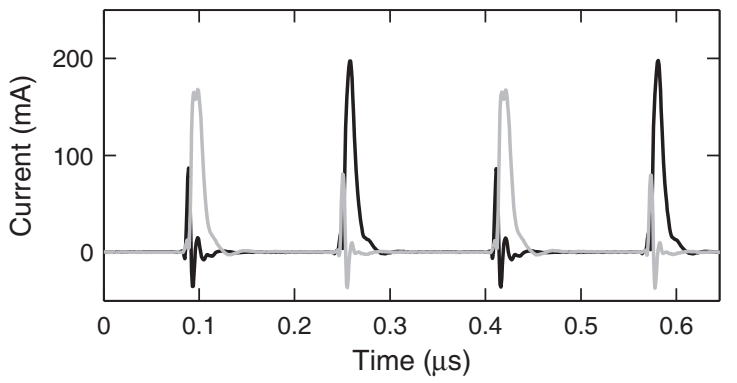

(b) Standard A \& B drive current

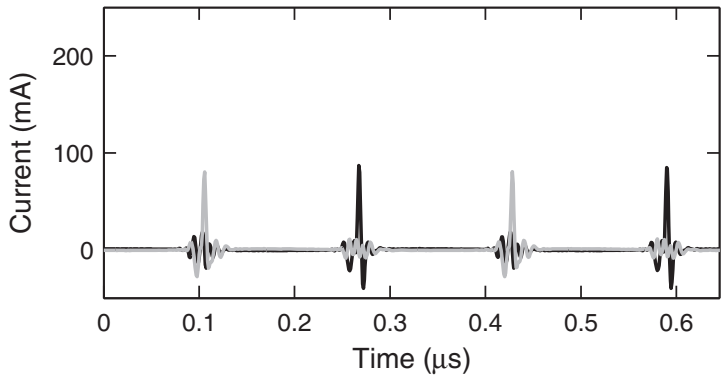

(d) Resonant A \& B drive current

Figure 5. Modulation waveforms and drive current for standard and resonant modulation at 3.1 MHz. In all plots, the response of channel A is traced in black, and channel B traced in grey.

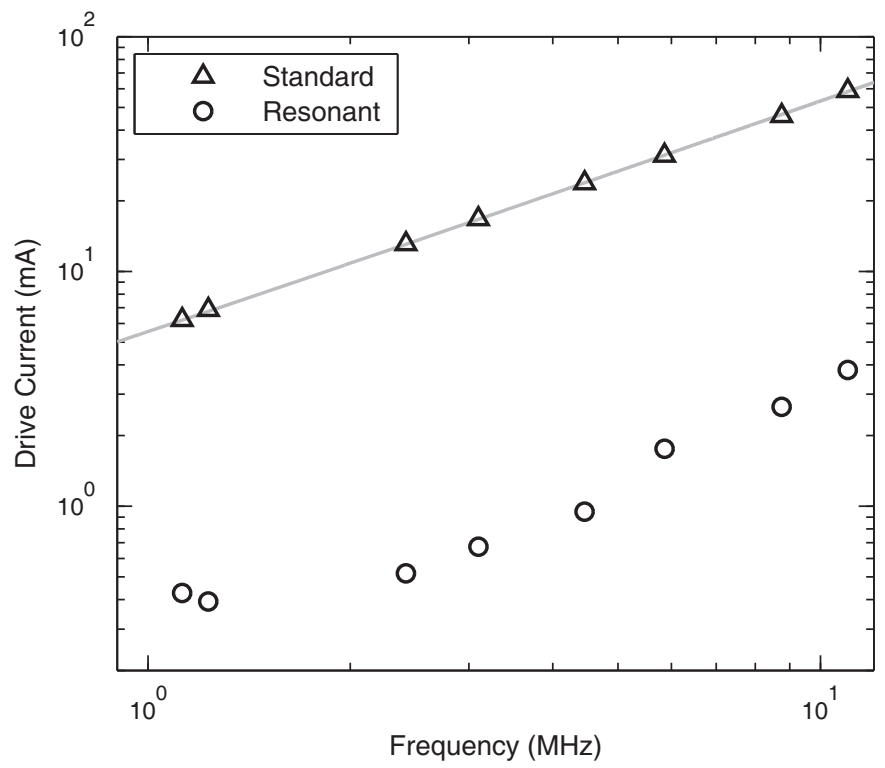

Figure 6. Average standard and resonant modulation drive current measurements for various frequencies from 1-11 MHz, illustrating power savings greater than an order of magnitude in each case. 


\subsection{Verifying Sensor Capacitance}

Some inconsistencies were noticed between the electrical measurement results and theoretical predictions. Firstly, in all resonant test cases the predicted and measured resonant frequencies differed beyond reasonable bounds of uncertainty; and secondly, when operating with standard modulation, the measured power did not match what was expected from equation 6. A possible explanation for this is that the actual cumulative modulation gate capacitance of the PMD $3 \mathrm{k}-\mathrm{S}$ sensor differs from the stated nominal value of $1.6 \mathrm{nF}$ per channel.

An indicative "actual" capacitance was calculated for each resonant test case by rearranging equation 8 and using the measured resonant frequencies and corresponding nominal inductance values. The calculated capacitance values (reported in table 1) are remarkably consistent with a mean value of $1.2 \mathrm{nF}$, even without taking into account the inductor tolerance levels $( \pm 5-10 \%)$, circuit damping effects, or any other uncertainties.

Table 1. Sensor modulation capacitance values calculated from the measured resonant frequencies and nominal inductance values.

\begin{tabular}{|c|c|c|}
\hline Inductor & Resonant Frequency $(\mathrm{MHz})$ & Capacitance $(\mathrm{nF})$ \\
\hline $33 \mu \mathrm{H} \pm 10 \%$ & 1.125 & 1.2 \\
$27 \mu \mathrm{H} \pm 10 \%$ & 1.23 & 1.2 \\
$8.2 \mu \mathrm{H} \pm 5 \%$ & 2.42 & 1.1 \\
$4.7 \mu \mathrm{H} \pm 5 \%$ & 3.10 & 1.1 \\
$2.2 \mu \mathrm{H} \pm 10 \%$ & 4.46 & 1.2 \\
$1.2 \mu \mathrm{H} \pm 5 \%$ & 5.86 & 1.2 \\
$560 \mathrm{nH} \pm 10 \%$ & 8.76 & 1.2 \\
$330 \mathrm{nH} \pm 10 \%$ & 10.975 & 1.3 \\
\hline
\end{tabular}

The modulation capacitance can also be empirically derived from a linear fit of the standard drive current measurements shown in figure 6. Using this approach yields a capacitance of $1.16 \pm 0.06 \mathrm{nF}$, which is comparable to the values listed in table 1 . These results support the notion that the actual modulation gate capacitance of this particular sensor is different to its stated nominal value.

\section{RANGE IMAGING PERFORMANCE}

With resonant modulation the electrical waveform shape is inherently different to that of the standard square waveform as evident in figure 5. While it is known that the shape of a pixels optical modulation response has an influence on range imaging performance, ${ }^{14}$ the effect of changing the electrical waveform shape is difficult to predict without prior knowledge of the pixels electrical-optical transfer function. Therefore, in order to compare performance between the standard and resonant operating modes, an experimental evaluation of phase measurement linearity and precision was carried out. Measurements were acquired at modulation frequencies of 1.23, 3.10, 5.86, 8.76 and $10.975 \mathrm{MHz}$; a subset of the resonant test cases listed in table 1.

A scene was set up consisting of a flat diffuse target placed at approximately $1 \mathrm{~m}$ from the camera. The relationship between measurement amplitude and precision is such that any difference in precision is more discernible at lower intensities (refer to equation 5). Therefore to obtain both low and high amplitude measurements half of the flat target was covered in a low reflectance material. Illumination was provided by a set of eight red laser diodes arranged in a circular configuration around a C-mount lens used to image the reflected modulated light onto the PMD sensor. These digitally driven lasers produced a relatively square $50 \%$ duty cycle amplitude modulated output containing higher order harmonic components. 


\subsection{Measurement Linearity}

To determine measurement linearity, the phase of the light source modulation was electronically swept through $0-2 \pi$ rad in relation to the sensor modulation signal, with images captured at $\pi / 50 \mathrm{rad}$ increments. This approach emulates the effect of moving the target away from the camera, whilst isolating the contamination due to harmonic interference between the modulation signals and minimising all other error sources. ${ }^{2}$ Traditional four-frame homodyne processing was used to generate a set of phase measurements at each phase offset. The measured values were then subtracted from the true phase values to reveal any systematic linearity error. In order to compute the average systematic error for each pixel, the phase sweep was repeated 100 times.

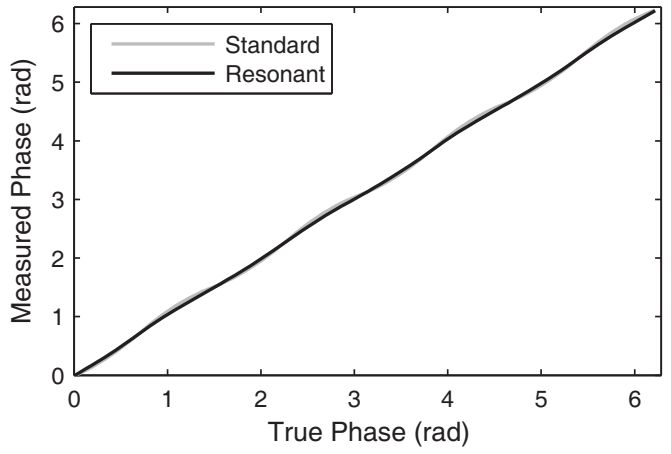

(a) True versus measured phase

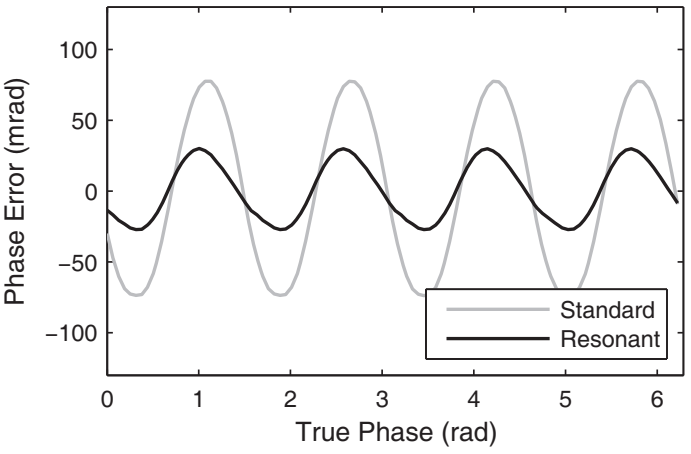

(b) Linearity error

Figure 7. Actual versus measured phase linearity measurements for a single pixel at a modulation frequency of $3.10 \mathrm{MHz}$ showing that the linearity error is reduced in the resonant mode.

The true versus average measured phase and resulting linearity error for a single pixel modulated at $3.10 \mathrm{MHz}$ for both standard and resonant operating modes is plotted in figure 7. In the standard configuration a typical cyclic linearity response ensued with the impact of aliased square wave harmonics resulting in an peak-to-peak error of $152 \mathrm{mrad}$. Although aliasing effects are still evident, resonant modulation exhibited a significantly lower peak-to-peak linearity error of $57 \mathrm{mrad}$, a reduction of $62 \%$ compared to the standard mode. Indeed, at all five test frequencies an improvement in measurement accuracy was observed as shown in table 2. These results indicate that the shape of a PMD pixels optical response with a resonant electrical modulation signal lies somewhere between sinusoidal and square with reasonably low amplitude harmonic components.

Table 2. Measured peak-to-peak linearity error for a single pixel at various test frequencies showing that resonant modulation provides improved linearity in all cases.

\begin{tabular}{|c|c|c|c|}
\hline \multirow{2}{*}{ Frequency $(\mathrm{MHz})$} & \multicolumn{2}{|c|}{ Peak-to-Peak Linearity Error (mrad) } & \multirow{2}{*}{ Percentage Reduction } \\
\cline { 2 - 3 } & Standard & Resonant & \\
\hline 1.23 & 160 & 64 & $60 \%$ \\
3.10 & 152 & 57 & $62 \%$ \\
5.86 & 142 & 52 & $63 \%$ \\
8.76 & 210 & 52 & $75 \%$ \\
10.975 & 227 & 51 & $77 \%$ \\
\hline
\end{tabular}

It must be noted that the measured linearity error using the standard operating mode at $10.975 \mathrm{MHz}$ and $8.76 \mathrm{MHz}$ was substantially higher than the approximate $150 \mathrm{mrad}$ peak-to-peak error that would normally be expected from the harmonic interaction of ideal square sensor and illumination modulation signals (see section 2.1). At these frequencies the electrical 
modulation waveforms exhibited larger harmonic content than anticipated (compared with an ideal square wave), which may have brought about the worse than expected response. Further investigation is required to establish the cause of this discrepancy.

\subsection{Measurement Precision}

The data used for determining phase linearity was also used to compare measurement precision between the standard and resonant configurations. Precision was determined by calculating the $1 \sigma$ standard deviation of phase values across 100 measurements. With precision being phase dependent for non-sinusoidal modulation signals due to aliased harmonics (as discussed in section 2.1), a value was calculated at each $\pi / 50$ rad phase step. The overall precision was found by taking the mean of the standard deviation values across the full $0-2 \pi$ rad phase sweep.

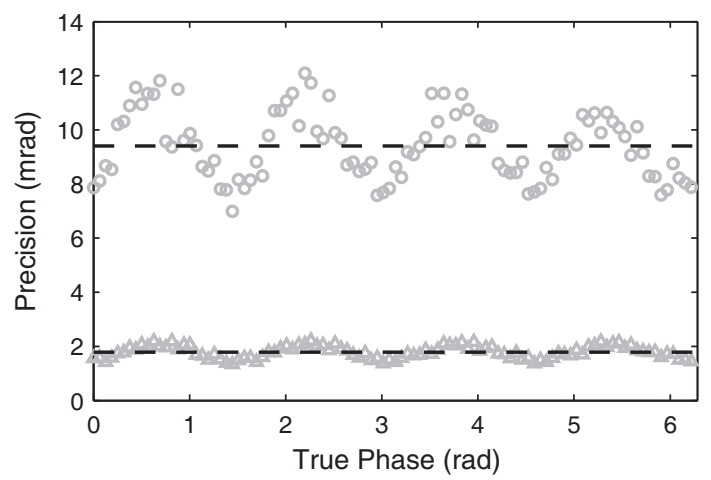

(a) Standard modulation

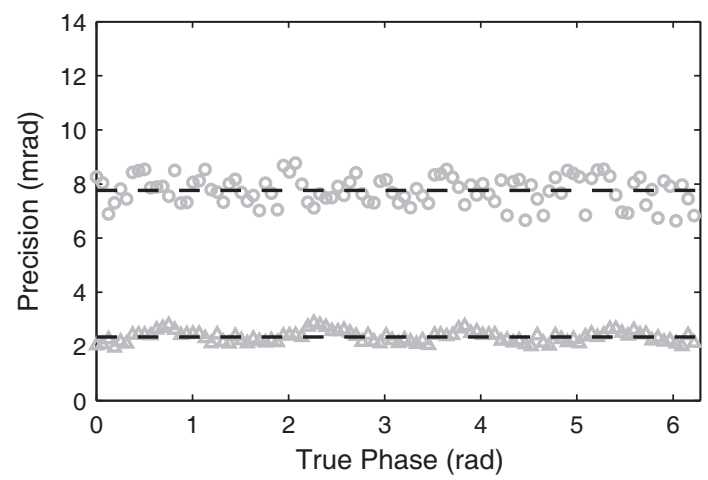

(b) Resonant modulation

Figure 8. Measurement precision versus phase at 3.10 MHz in both standard and resonant operating modes for both a bright pixel $(\triangle)$, and a dark pixel $(0)$. The broken line shows the average precision value across $0-2 \pi \mathrm{rad}$.

In order to better observe any difference in precision between the two configurations, values were calculated for both a bright pixel and a dark pixel on the target. Precision values for standard and resonant modulation at 3.10 $\mathrm{MHz}$ are plotted in figure 8, which shows further evidence of reduced harmonic interference in the resonant mode. This is particularly apparent for the dark pixel where figure 8(a) shows a distinct cyclic trend that is not as perceptible in figure 8(b).

Table 3. Average measured bright and dark pixel precision values for both standard and resonant modulation.

\begin{tabular}{|c|c|c|c|c|}
\hline \multirow{2}{*}{ Frequency $(\mathrm{MHz})$} & \multicolumn{2}{|c|}{ Bright Pixel Precision (mrad) } & \multicolumn{2}{c|}{ Dark Pixel Precision (mrad) } \\
\cline { 2 - 5 } & Standard & Resonant & Standard & Resonant \\
\hline 1.23 & 1.8 & 2.0 & 9.6 & 13.0 \\
3.10 & 1.8 & 2.3 & 9.4 & 7.8 \\
5.86 & 1.7 & 1.9 & 9.1 & 8.2 \\
8.76 & 1.7 & 2.0 & 9.3 & 8.6 \\
10.975 & 1.7 & 1.6 & 9.2 & 8.1 \\
\hline
\end{tabular}

The mean precision results indicate comparable performance for the bright pixel with values of 2.3 mrad using resonant modulation compared to $1.8 \mathrm{mrad}$ in the standard mode. For the dark pixel a slight precision improvement was observed, with $7.8 \mathrm{mrad}$ and $9.4 \mathrm{mrad}$ for resonant and standard modulation respectively. Similar results were recorded across most measurement frequencies, as reported in table 3, with the exception of $1.23 \mathrm{MHz}$ where the dark pixel precision was worse in the resonant configuration. 
These results are somewhat surprising. Considering the $3.10 \mathrm{MHz}$ case, by inspection the peak-to-peak amplitudes of the measured standard (square) and resonant (sinusoidal) electrical modulation waveforms are similar (comparing figures 5(a) and 5(c)). Intuitively, this means that the amplitude of the fundamental frequency component is smaller in the resonant mode, which if translated to the optical domain would lead to a reduction in demodulation contrast and thus poorer precision; however the results seem to be contradictory showing a slight improvement. An evaluation of the sensors optical response may help reveal an explanation for this, but further investigation is beyond the scope of this paper and is left for future work.

\section{CONCLUSION}

In a time-of-flight range imaging camera, sensor gain modulation can contribute a significant portion of the total image sensor power. It has been demonstrated that by augmenting the modulation circuitry to form a distributed resonant tank circuit, considerable power savings can be achieved. At all test frequencies, the measured power due to resonant sensor modulation was smaller than the standard (square) modulation power consumption by more than an order of magnitude. In terms of range imaging performance, the inherent sinusoidal shape of the resonant electrical waveforms led to an improvement in phase (distance) measurement linearity, while comparable measurement precision was observed between the two operating modes. In summary, these results indicate that resonant sensor modulation is a viable power saving strategy as measurement performance was not significantly compromised.

\section{ACKNOWLEDGMENTS}

Richard Conroy acknowledges the support of a New Zealand Tertiary Education Commission Top Achiever Doctoral Scholarship. This research is sponsored by the University of Waikato Strategic Investment Fund.

\section{REFERENCES}

[1] Oggier, T., Lehmann, M., Kaufmann, R., Schweizer, M., Richter, M., Metzler, P., Lang, G., Lustenberger, F., and Blanc, N., "An all-solid-state optical range camera for 3D real-time imaging with sub-centimeter depth resolution (SwissRanger)," Proc. SPIE 5249, 534-545 (2004).

[2] Payne, A. D., Dorrington, A. A., Cree, M. J., and Carnegie, D. A., "Improved measurement linearity and precision for AMCW time-of-flight range imaging cameras," Appl. Opt. 49(23), 4392-4403 (2010).

[3] Buttgen, B. and Seitz, P., "Robust optical time-of-flight range imaging based on smart pixel structures," IEEE Trans. Circuits Syst. I, Reg. Papers 55(6), 1512-1525 (2008).

[4] Gokturk, S., Yalcin, H., and Bamji, C., "A time-of-flight depth sensor - system description, issues and solutions," Proc. 2004 IEEE Conf. Computer Vision and Pattern Recognition Workshop, 35 (2004).

[5] PMD Technologies GmbH, "PhotonICs PMD 3k-S 3D video sensor array with active SBI $64 \times 48$ pixels datasheet (Revision 1.4)," http://www.pmdtec.com (2005).

[6] PMD Technologies GmbH, "PhotonICs PMD 19K high resolution 3D video sensor array $160 \times 120$ pixels datasheet (Revision 2.4)," http://www.pmdtec.com (2006).

[7] Athas, W. C., Tzartzanis, N., Svensson, L. J., and Peterson, L., "Low-power microprocessor based on resonant energy," IEEE J. Solid-State Circuits 32(11), 1693-1701 (1997).

[8] Drake, A. J., Nowka, K. J., Nguyen, T. Y., Burns, J. L., and Brown, R. B., "Resonant clocking using distributed parasitic capacitance," IEEE J. Solid-State Circuits 39(9), 1520-1528 (2004).

[9] Hansson, M., Mesgarzadeh, B., and Alvandpour, A., "1.56 GHz on-chip resonant clocking in 130nm CMOS," Proc. Custom Integrated Circuits Conference, 241-244 (2006).

[10] Chan, S. C., Shepard, K. L., and Restle, P. J., "Distributed differential oscillators for global clock networks," IEEE J. Solid-State Circuits 41(9), 2083-2094 (2006).

[11] Sathe, V. S., Kao, J. C., and Papaefthymiou, M. C., "Resonant-clock latch-based design," IEEE J. Solid-State Circuits 43(4), 864-873 (2008).

[12] Conroy, R. M., Dorrington, A. A., Künnemeyer, R., and Cree, M. J., "Range imager performance comparison in homodyne and heterodyne operating modes," Proc. SPIE 7239, 723905 (2009).

[13] Intersil Corp., "EL7158 ultra-high current pin driver datasheet," http://www.intersil.com/data/fn/fn7349.pdf (2007).

[14] Payne, A. D., Dorrington, A. A., Cree, M. J., and Carnegie, D. A., "Characterization of modulated time-of-flight range image sensors," Proc. SPIE 7239, 723904 (2009). 